\title{
The association between household poverty rates and tuberculosis case notification rates in Cambodia, 2010
}

\author{
Man Kai Wong, ${ }^{a}$ Rajendra-Prasad Yadav, ${ }^{a}$ Nobuyuku Nishikiorib and Mao Tan Eang ${ }^{c}$ \\ Correspondence to Man Kai Wong (e-mail: Alyssa.mkw@gmail.com).
}

Introduction: Poverty is a risk factor for tuberculosis (TB); it increases the risk of infection and active disease but limits diagnostic opportunities. The role of poverty in the stagnant case detection in Cambodia is unclear. This study aims to assess the relationship between district household poverty rates and sputum-positive TB case notification rates (CNR) in Cambodia in 2010.

Methods: Poisson regression models were used to calculate the relative risk of new sputum-positive TB CNR for Operational Districts (ODs) with different poverty rates using data from the National Centre for Tuberculosis and Leprosy Control and the National Committee for SubNational Democratic Development. Models were adjusted for other major covariates and a geographical information system was used to examine the spatial distribution of these covariates in the country.

Results: The univariate model showed a positive association between household poverty rates and sputum-positive TB CNRs. However, in multivariate models, after adjusting for major covariates, household poverty rates showed a significantly negative association with sputum-positive TB CNRs (relative risk $[R R]=0.95$ per $5 \%$ increase in poverty rate). The negative association was stronger among males than females ( $R R=0.93$ versus 0.96 per $5 \%$ increase in poverty rate). Similar spatial patterns were observed between household poverty rates and other covariates, particularly OD population density.

Conclusion: Household poverty rate is associated with a decrease in sputum-positive TB CNR in Cambodia, particularly in men. The potential of combining surveillance data and socioeconomic variables should be explored further to provide more insights for TB control programme planning.

$\mathrm{T}$

he links between poverty and tuberculosis (TB) cases have been documented for decades in many parts of the world. ${ }^{1-3}$ While poverty may lead to higher case notification rates (CNRs) due to the higher prevalence of active TB cases among poorer populations, it is also thought that poverty may lower CNRs as a result of reduced access to TB diagnosis and poor diagnostic capacity in such populations.

Cambodia is currently identified by the World Health Organization (WHO) as one of the 22 countries in the world that carry the highest TB burden with approximately 61000 new TB cases per year and an incidence rate of 424 cases per 100000 population in $2010 .{ }^{4}$ It is estimated that $64 \%$ of Cambodians are infected with latent or active TB, and a substantial number of cases remain undetected. ${ }^{5}$ In 1994 , WHO's global TB control strategy (the DOTS strategy) was introduced and has reached almost 100\% coverage and $90 \%$ treatment success rate for all forms of TB. However, the case detection rate (CDR) for all forms of TB has stagnated at around $65 \%$. Since 2005 , the country began to explore active case finding (ACF) initiatives to increase overall case notification in which the providers visit target populations in teams and use screening tools to identify TB suspects, followed by diagnostic tools to identify the TB patients on the spot. ${ }^{6}$ The ACF initiatives try to complement case-finding through passive casefinding efforts, wherein people with symptoms visit health facilities and the health workers use screening tools to identify TB suspects.

Given the strong links between poverty and TB burden, many efforts had been made in developing countries to target poor and vulnerable groups. ${ }^{7}$ However, TB incidence is only estimated at the national level, while

\footnotetext{
World Health Organization Representative Office, Phnom Penh, Cambodia.

World Health Organization Regional Office for the Western Pacific, Manila, Philippines.

National Centre for Tuberculosis and Leprosy Control, Ministry of Health, Phnom Penh, Cambodia.

Submitted: 17 January 2013; Published: 31 March 2013

doi: 10.5365/wpsar.2013.4.1.002
} 
the number of cases diagnosed and CNRs are often the only measures available at provincial and district levels. The CNR does not reflect the actual number of active TB cases but rather the number of cases diagnosed and put on treatment; the CNR can be greatly affected by access to services, which, in turn, is often affected by poverty. Provincial and district TB control authorities often rely heavily on TB case notification data to find the missing cases, but they may not be aware of the population to target within their geographical areas to maximize their efforts.

Using a cross-sectional analysis approach, this study aimed to assess the link between household poverty level and sputum-positive TB CNR in Cambodia in 2010. The study also explored the potential of using a geographical information system (GIS) to characterize the spatial distribution of poverty and other TB risk factors with the distribution of TB. ${ }^{8-10}$

\section{METHODS}

We performed cross-sectional ecological analyses using operational districts (ODs) as the unit of analysis. $A n O D$ is the basic organizational and reporting unit in the Cambodian health system, providing services through health centres and district referral hospitals. There are 77 ODs in Cambodia, spread over 24 provinces, each serving an average population of 180000 (range: 35 500-525 500). The ODs are formed by combining several communes, which are the lowest level administrative units in Cambodia each consisting of three to 30 villages. According to the 2008 census, there were 1621 communes and 14073 villages in Cambodia.

\section{Data source}

The number of newly diagnosed sputum-positive TB cases (including new and previously treated TB cases) in 2010 was obtained from the National TB Registry maintained by the National Centre for Tuberculosis and Leprosy Control(CENAT). The registry containsinformation on the number of different types of TB cases diagnosed, put on treatment and reported by all OD TB coordinators. CNRs were computed based on the number of cases and the population of the ODs projected for the year, based on figures from the latest census (2008). Case count by age group was only available for new sputum- positive TB cases (not including previously treated TB cases); therefore, CNRs for different age groups were not computed.

Household poverty data were obtained from the Commune Database maintained by the National Committee for SubNational Democratic Development, which contains core information regarding demographic, socioeconomic and physical assets of each commune in Cambodia ( $n=1620$ ). These data are collected by village chiefs and commune clerks annually and are compiled at the commune level. Household poverty rates (\% of population living at or below poverty line) for each commune were derived based on the poverty line and the per capita consumption expenditure per day for the year. We estimated the OD poverty rates by spatially matching communes with ODs and averaging all the commune poverty rates in the same OD.

Population density in each OD was calculated by dividing the population projected for 2010 , by the area of each OD (in square kilometres), data that were obtained from the WHO Western Pacific Regional Office MapServer for Public Health Mapping.

Other factors potentially associated with TB disease burden were selected from the Cambodia Demographic and Health Surveys (DHS) that are nationally and regionally representative household surveys that provide a wide range of monitoring and impact evaluation indicators. Because subregional estimates in the DHS surveys were not based on representative samples, we used the regional (provincial) estimates in this study and applied the same proportions to all the ODs in the same province.

\section{Patient diagnostic rate}

Patient diagnostic rate (PDR), an indicator to assess case detection, is the rate at which prevalent cases are detected by control programmes and is calculated using the number of reported cases per 100000 per year divided by the prevalence per 100 000. ${ }^{11}$ In this study, prevalence data were obtained from the Second National TB Prevalence Survey (2011) conducted by CENAT. ${ }^{12}$ The denominator represents the population that had TB at the time and the numerator represents those actually detected and notified by the National TB Control Programme. 


\section{Table 1. Categorization of values of the covariates}

\begin{tabular}{lccc}
\hline \multicolumn{1}{c}{ Categories } & Low & Medium & High \\
\hline $\begin{array}{l}\text { Operational districts population density }\left(\text { population } / \mathrm{km}^{2}\right) \\
\begin{array}{l}\text { Distance to health care facility }(\% \text { of women who perceived distance as a } \\
\text { problem in accessing health care) }\end{array}\end{array}$ & $<100$ & $100-250$ & $\geq 250$ \\
All basic vaccination coverage $(\%$ population vaccinated) & $<30$ & $30-50$ & $\geq 50$ \\
HIV prevalence (\% adults 15-49 who have HIV) & $<70$ & $70-80$ & $\geq 80$ \\
\hline
\end{tabular}

\section{GIS spatial characterization}

Data on all new and previously treated sputum-positive TB cases and OD household poverty rates were linked to their respective geo-coded OD data using the join attrition function in Arc Map GIS software V.9.3.1 (Environmental System Research Institute, Redlands, CA, USA). Similarly, OD population density, all basic vaccination coverage, distance to health care facilities and HIV prevalence were also mapped with sputumpositive TB CNR using classifications that are the same as those defined in this study.

\section{Statistical analysis}

Using multiple Poisson regression models, the logarithm of the number of new sputum-positive TB cases notified in each $O D$ was modelled as a function of household poverty with the logarithm of the OD population as an offset. The household poverty rates were transformed into both continuous and categorical variables in increments of $5 \%$. Relative risks (RR) of new smear-positive TB case notification rates were estimated from the models using the lowest poverty category as the referent group. A univariate model was used to assess the effect of household poverty rates on new sputum-positive TB CNRs, followed by a multivariate model adjusting for (1) OD population density; (2) HIV prevalence; (3) distance to health facility (\% of women who perceived distance to health facility as a problem in accessing health care); and (4) all basic vaccination coverage (proportion vaccinated). All covariates were identified a priori and included in the model as tertile discrete continuous variables (Table 1). SAS 9.2 was used for all analyses.

\section{RESULTS}

All 77 ODs were included in the continuous and categorical analyses. Table 2 shows the summary characteristics of ODs in each poverty category.

The continuous univariate model showed an overall positive association between household poverty rates and sputum-positive TB CNRs (Table 3). A 5\% increase in household poverty rates was associated with a $2 \%$ increase in TB CNRs (RR $=1.02 ; 95 \%$ confidence interval $[\mathrm{Cl}]=1.00,1.03)$. A significant association was seen among females $(\mathrm{RR}=1.03 ; 95 \% \mathrm{Cl}=1.01$, 1.04 per $5 \%$ increase in poverty rate), while the association was nil for males $(R R=1.00)$. After adjusting for OD population density, distance to health facility, HIV prevalence and all basic vaccination coverage, the continuous adjusted model showed an overall negative association between household poverty rates and sputum-positive TB CNRs. The model suggested that a $5 \%$ increase in household poverty rates was associated with a $5 \%$ decrease in sputum-positive TB CNRs $(R R=0.95 ; 95 \% \mathrm{Cl}=0.92,0.96)$. A stronger negative association was seen among males $(\mathrm{RR}=0.93$; $95 \% \mathrm{Cl}=0.91,0.96$ per $5 \%$ increase in poverty rate) than females ( $R R=0.96 ; 95 \% \mathrm{Cl}=0.93$, 0.99 per $5 \%$ increase in poverty rate).

In the categorical analysis (Figure 1), ODs with $15 \%-20 \%$ of the population living below the poverty line were associated with the highest sputum-positive TB CNRs ( $R R=1.35 ; 95 \% \mathrm{Cl}=1.23,1.48$ per $5 \%$ increase in poverty rate) after adjusting for OD population density, distance to health care facility, vaccination coverage and HIV prevalence. Subsequent increase in poverty rates resulted in lower RRs. A similar trend of 
Table 2. Characteristics of operational districts in each poverty category

\begin{tabular}{|c|c|c|c|c|c|c|}
\hline \multirow{3}{*}{$\begin{array}{l}\text { Household poverty (\% below poverty line) } \\
\text { Number of ODs (N) }\end{array}$} & \multicolumn{2}{|c|}{$<15$} & \multicolumn{2}{|c|}{$15-19$} & \multicolumn{2}{|c|}{$20-24$} \\
\hline & \multicolumn{2}{|c|}{6} & \multicolumn{2}{|c|}{5} & \multicolumn{2}{|c|}{15} \\
\hline & Mean & SD & Mean & SD & Mean & SD \\
\hline OD populations & 242309.2 & 106929.2 & 110799.2 & 41183.1 & 184348.7 & 68373.3 \\
\hline Population density (people/km²) & 4615.2 & 6950.9 & 245.4 & 113.2 & 251.3 & 114.8 \\
\hline \multicolumn{7}{|l|}{$\begin{array}{l}\text { Number of new sputum-positive TB cases in } 2010 \\
\text { (new and relapsed) }\end{array}$} \\
\hline Male & 104.8 & 27.8 & 84.8 & 31.5 & 115.7 & 56.0 \\
\hline Female & 74.2 & 31.5 & 75.8 & 35.0 & 111.9 & 68.1 \\
\hline Relapsed (male and female) & 95.0 & 70.1 & 4.8 & 4.1 & 10.4 & 12.0 \\
\hline Total & 274.0 & 69.9 & 165.4 & 66.9 & 237.9 & 127.8 \\
\hline Case notification rate (per 100000 population) & 126.3 & 39.8 & 151.2 & 40.1 & 125.4 & 53.2 \\
\hline HIV prevalence (adults $15-49$ years) & 1.2 & 0.7 & 0.5 & 0.3 & 0.5 & 0.3 \\
\hline All basic vaccination coverage (\%) & 81.8 & 3.7 & 69.2 & 10.8 & 75.7 & 10.7 \\
\hline Distance to health facilities (\% perceived as problem) & 22.1 & 8.7 & 23.5 & 17.4 & 35.1 & 20.6 \\
\hline Household poverty (\% below poverty line) & \multicolumn{2}{|c|}{$25-29$} & \multicolumn{2}{|c|}{$30-34$} & \multicolumn{2}{|c|}{$\geq 35$} \\
\hline \multirow[t]{2}{*}{ Number of ODs (N) } & \multicolumn{2}{|c|}{23} & \multicolumn{2}{|c|}{19} & \multicolumn{2}{|c|}{9} \\
\hline & Mean & SD & Mean & SD & Mean & SD \\
\hline OD populations & 173205.0 & 72311.5 & 193672.0 & 112518.2 & 178326.7 & 63170.8 \\
\hline Population density (people/km²) & 183.7 & 95.1 & 116.4 & 126.7 & 31.2 & 24.5 \\
\hline \multicolumn{7}{|l|}{$\begin{array}{l}\text { Number of new sputum-positive TB cases in } 2010 \\
\text { (new and relapsed) }\end{array}$} \\
\hline Male & 110.5 & 69.4 & 137.1 & 69.1 & 113.3 & 64.1 \\
\hline Female & 90.6 & 62.3 & 127.6 & 76.8 & 87.0 & 50.8 \\
\hline Relapsed (male and female) & 10.1 & 25.2 & 10.5 & 16.3 & 7.4 & 11.9 \\
\hline Total & 211.3 & 129.7 & 275.2 & 147.3 & 207.8 & 117.3 \\
\hline Case notification rate (per 100000 population) & 119.0 & 51.3 & 148.8 & 38.9 & 115.2 & 58.4 \\
\hline HIV prevalence (adults $15-49$ years) & 0.5 & 0.2 & 0.5 & 0.3 & 0.3 & 0.3 \\
\hline All basic vaccination coverage $(\%)$ & 82.2 & 6.0 & 81.8 & 8.0 & 64.4 & 21.6 \\
\hline Distance to health facilities (\% perceived as problem) & 35.0 & 14.5 & 42.6 & 11.8 & 56.3 & 12.0 \\
\hline
\end{tabular}

ODs - operational district; SD - standard deviation

Note: HIV prevalence was averaged based on provincial-level data from 2005 DHS database. All basic vaccination coverage, distance to health facilities and education was averaged based on provincial-level data from 2010 DHS database. All basic vaccinations include BCG, measles and three doses each of tetravalent or pentavalent and polio vaccine.

decrease in RRs across the poverty level was observed in both males and females, although the RR of sputumpositive TB CNR was higher for females than males across all poverty categories.

The patient diagnostic rate (PDR) for the overall population was 0.5 ; the PDR for males was much lower than for females ( 0.37 versus 0.77 ) (Figure 2 ).

\section{Spatial characterization}

Although the highest household poverty rates are found in the northeastern part of Cambodia, the region accounts for some of the lowest sputum-positive TB CNRs (Figure 3). The same region also has the lowest vaccination coverage and the worst physical barrier of distance to health care facilities (Figure 4). The southern part of the country, which is less poor than the other regions, showed much higher sputumpositive TB CNRs. There were notable links between the map of OD population density (the top left of Figure 4) and the map of household poverty rate (Figure 3) where ODs with higher population density were often areas of lower poverty. Clusters of ODs with a high HIV prevalence were observed near the central region and along the coastal regions. 
Table 3. Effect of household poverty on tuberculosis case notification using a continuous model

\begin{tabular}{lcccc}
\hline & Univariate RR $(95 \% \mathrm{Cl})$ & $P$-value & Adjusted RR $(95 \% \mathrm{Cl})$ & $P$-value \\
\hline Overall & $1.02(1.00-1.03)$ & 0.0045 & $0.95(0.92-0.96)$ & $<0.0001$ \\
Male & $1.00(0.99-1.02)$ & 0.4966 & $0.93(0.91-0.96)$ & $<0.0001$ \\
Female & $1.03(1.01-1.04)$ & 0.0006 & $0.96(0.93-0.99)$ & 0.0030 \\
\hline
\end{tabular}

Note: All relative risks (RR) shown correspond to a $5 \%$ increase in the proportion of population living at or below the poverty level. Adjusted RR: adjusted for operational district (OD) population density, distance to health care facility, HIV prevalence and all basic vaccination coverage. All covariates were significantly associated with sputum-positive TB case notification rates $(C N R)(P<0.0001)$. Distance to health facility (\% of women who perceived it as problem for accessing health care facility) and all basic vaccine coverage was positively associated with sputum-positive TB CNR, whereas OD population density and HIV prevalence was negatively associated with sputum-positive TB CNR.

Figure 1. Relative risk for sputum-positive tuberculosis case notification in different household poverty levels, adjusted for operational district population density, distance to health care facility, vaccination coverage and HIV prevalence*
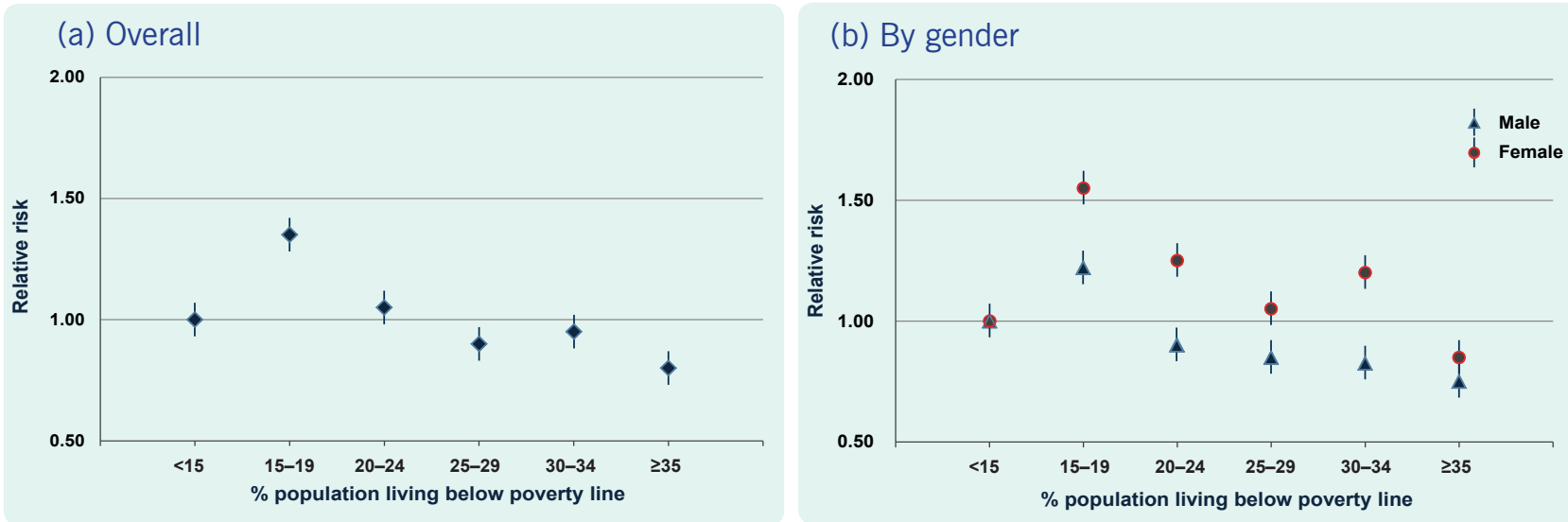

The lines above and below each point denote $95 \%$ confidence intervals. Operational districts with < $15 \%$ population living at or below poverty line was the referent group.

\section{DISCUSSION}

Our findings suggest that there was an overall negative association between household poverty and sputumpositive TB CNR in Cambodia in 2010 after adjustment for covariates like population density, distance to health care facilities, all basic vaccination coverage and HIV prevalence. The negative association was more apparent in males than females. Without adjusting for these variables, household poverty was positively associated with sputum-positive TB CNR. Given the widely documented positive associations between poverty and TB disease burden, ${ }^{13,14}$ we have several possible explanations for the differing findings in our study.

TB case notification (i.e. number of cases notified per 100000 population per year) is dependent on the actual prevalence and incidence of the disease, as well as the level of case detection (i.e. proportion of incident cases put on treatment by the national programme).
Although the poverty rate may be positively associated with TB prevalence and incidence rates, it may not be positively associated with CNR if CDR is low. CDR (and hence CNR) could be low due to poor geographical access to TB care, high costs associated with seeking care and poor awareness about TB disease and TB services.

In Cambodia and in most countries of the world, TB prevalence among males is significantly higher than among females. ${ }^{15}$ The latest prevalence survey in Cambodia indicated that the male-to-female ratio of smear-positive cases was $1.82 .{ }^{12}$ However, PDRs calculated using data from the same survey indicated that females were more likely to have been diagnosed, treated and reported than males by the programme. This matches with the findings of our study results, which illustrates that the reduction in sputum-positive TB CNR among females was not as significant as among males as poverty increases (RR of 0.96 versus 0.93 per $5 \%$ increase in poverty 
Figure 2. Prevalence of smear-positive tuberculosis, ${ }^{12}$ annual case notification rate and calculated patient diagnostic rate in Cambodia, 2011

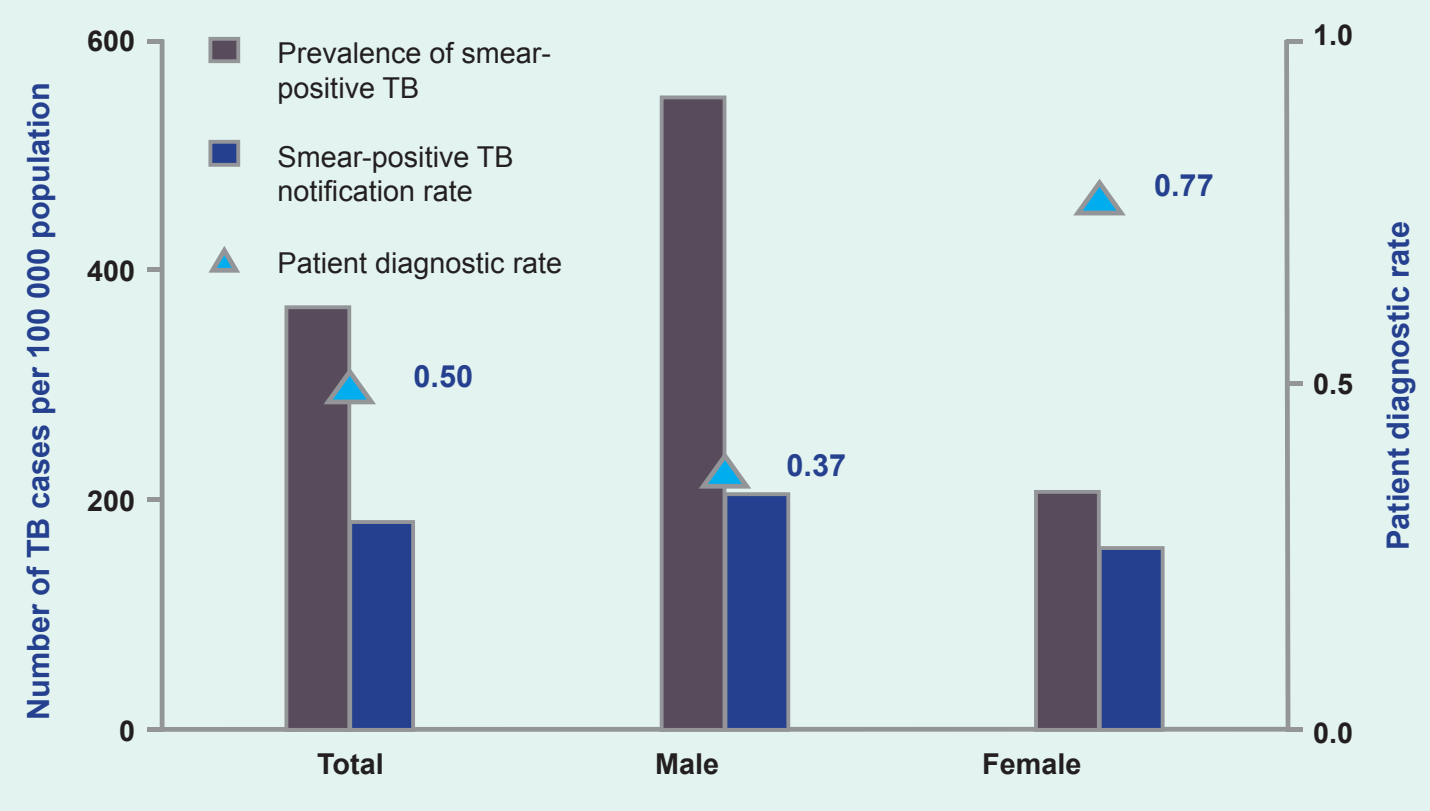

Figure 3. Map of smear-positive tuberculosis case notification rates with operational districts household poverty levels, Cambodia, 2010

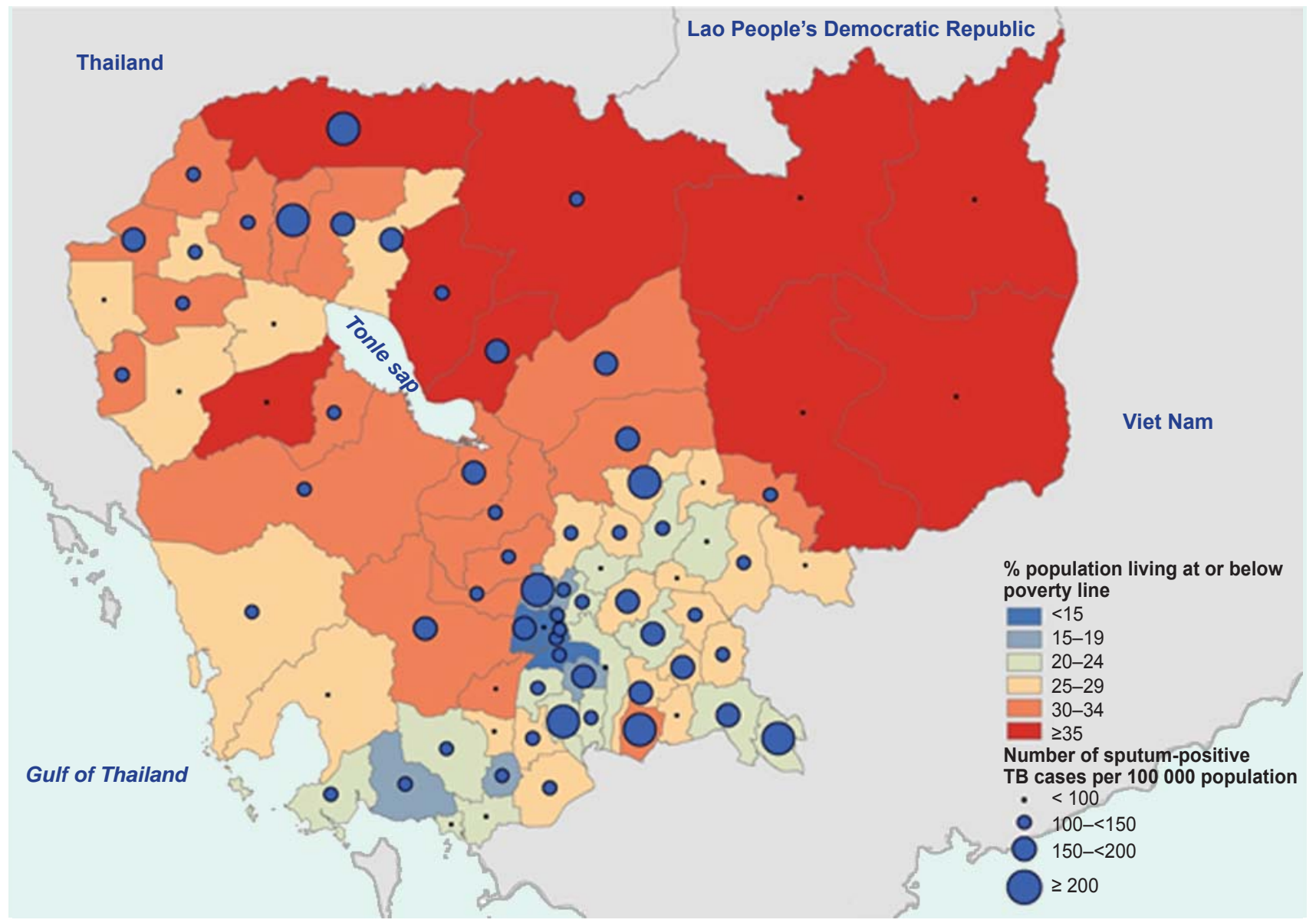

Source: Data from the National Committee for SubNational Democratic Development, World Food Programme, National Institute of Statisitics and National Centre for Tuberculosis and Leprosy Council, Cambodia 
Figure 4. Map of tuberculosis risk factor distribution with sputum-positive tuberculosis case notification rates in Cambodia, 2010

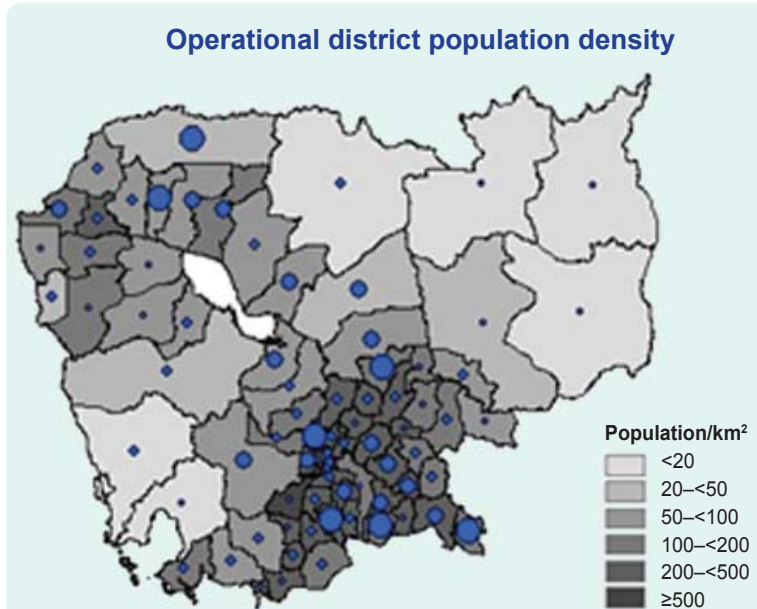

Distance to health care facilities

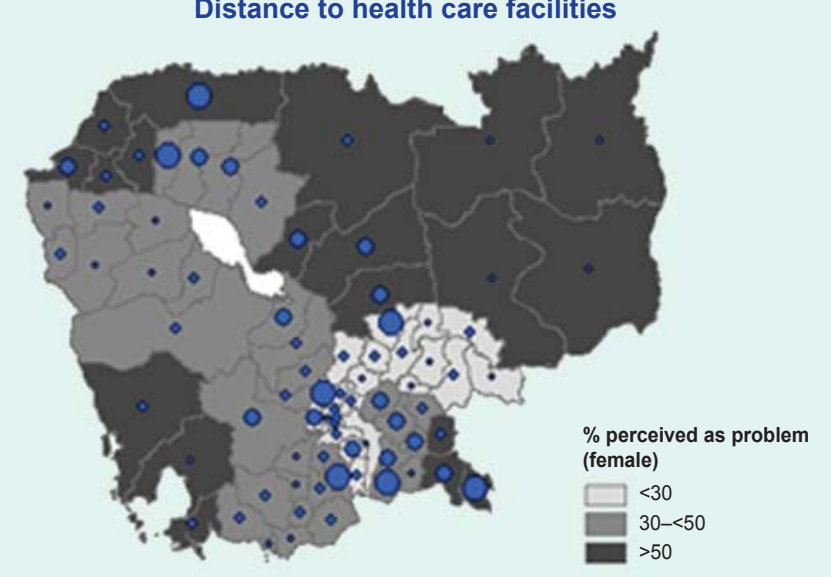

All basic vaccination coverage

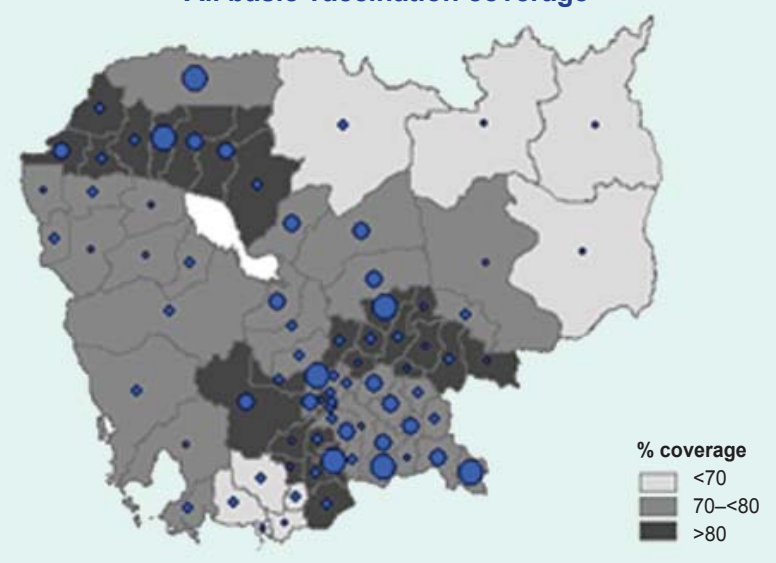

HIV prevalence

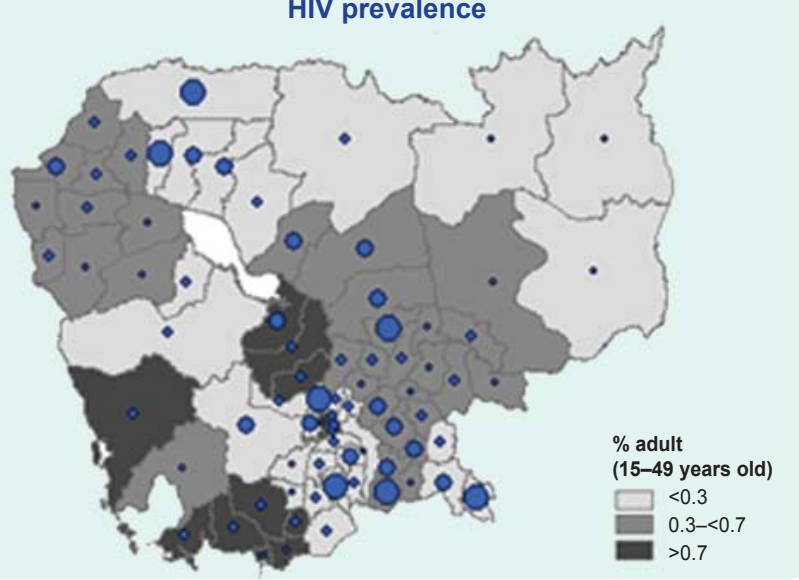

Note: Size of point corresponds to case notification rates, see Figure 2 map legend.

rate). Given that the negative association is stronger in males than in females, a greater proportion of males with active TB disease may remain undetected, especially in higher poverty regions. This could be driven by the difference in health-seeking behaviours between males and females since females tend to visit government health care facilities more often as a result of their closer association with these facilities during pregnancy and childbirth. Males may be seeking care in private clinics or may be more negligent of their symptoms and diseases compared to females.

A GIS provided an effective platform for the convergence of disease-specific information in this study. It allowed for visual assessment of the distribution of TB risk factors and TB CNRs, revealing trends and interrelationships that would be more difficult to discover in tabular format. In the long-term, GIS data should be used by policy-makers to easily visualize problems in relation to existing health services and socio-demographic features so that resources can be geo-targeted to achieve maximal health outcomes.

A major limitation of this study is the reliance on ecological-population-level data that are subject to ecological fallacy and Modifiable Areal Unit Problem. Exposure levels represent averages for each population group in this study; thus, findings between average exposure and TB case notification in this study do not imply that such a relationship would be present at the individual level. The association found in one aggregation level may change if the underlying data are aggregated differently. Using ODs as the unit of analysis may not give accurate results as these districts are too large and TB and poverty rates are heterogeneously distributed within ODs. In addition, it is difficult to control for potential confounders and to establish a correct temporal sequence between the exposure and outcome. The finding from 
this study may serve as preliminary investigation to stimulate additional research.

Given the widely studied links between age and TB risk, the inability to adjust for age distribution was also a limitation of our model. Age-specific CNRs were not available in an aggregated electronic database and were therefore excluded as outcome variables in our analysis. In addition, we could not adjust for OD age distribution because ODs are only used by the health ministry, while census data are collected at the provincial level. The validity of measurements is the other major limitation in this study. Household poverty rates were calculated based on standardized survey instruments administered by different village chiefs and commune clerks. It is not known whether the instruments were validated or if the survey responses were confirmed by a second representative. OD household poverty rates were calculated by averaging all the administrative district poverty rates, some of which had a wide variance. Furthermore, all covariate measures (except population density) were obtained from the DHS survey, which was only representative at the provincial level, not the OD level. ODs in the same province were assumed to have the same HIV prevalence, vaccination coverage and access problem, which may not accurately reflect the level of variance among all ODs. Distance to health care facilities was a measure of perceived barrier among women; it was not an actual measure of distance and may not reflect the experience of men. HIV prevalence data were extracted from the 2005 DHS survey, but other risk factors were extracted from the 2010 DHS survey. This is because HIV prevalence testing was not conducted in the 2010 DHS survey. While HIV prevalence in Cambodia is relatively low, we did observe a slight decrease in national HIV prevalence from 2005 to 2010. However, the extent to which this may have affected our results is unknown.

Although historically TB has been associated with poverty, few analytical studies from developing countries have tried to quantify the relative impact of poverty on TB case notification rates, explore the causal mechanism underlying this association and help programmes to geo-target areas with high poverty rates. This study is an example of using routinely collected data for research purposes. Despite the limitations inherent in these data sources, the findings are still valuable as they enhance our knowledge of disease control at a district level.
Further studies are still needed to identify possible explanations for the observed association in Cambodia. These studies should strive to conduct more disaggregated analysis using smaller geographical units, provided better data on poverty rate and CNR are available. The results of such targeted studies will better reflect the true determinants of TB burden and guide the National TB Programme to implement more effective TB control interventions.

\section{Conflicts of interest}

None declared.

\section{Funding}

None.

\section{Acknowledgements}

We would like to thank the Cambodia National Centre for Tuberculosis and Leprosy Control for providing case notification and prevalence data and the World Food Programme (Cambodia) staff for spatially matching communes with operational (health) districts.

\section{References:}

1. Grange J, Zumla A. Tuberculosis and the poverty-disease cycle. Journal of the Royal Society of Medicine, 1999, 92:105-107. pmid:10396250

2. Grange J, Story A, Zumla A. Tuberculosis in disadvantaged groups. Current Opinion in Pulmonary Medicine, 2001, 7:160-164. doi:10.1097/00063198-200105000-00008 pmid:11371772

3. Kearney MT et al. Tuberculosis and poverty. BMJ (Clinical Research Ed.), 1993, 307:1143. doi:10.1136/bmj.307.6912.1143 pmid:8110253

4. Global tuberculosis report 2012. Geneva, World Health Organization, 2012 (http://www.who.int/tb/publications/global_ report/gtbr12_main.pdf, accessed 30 September 2012).

5. Tuberculosis Profile Cambodia, 2009. Washington, DC, United States Agency for International Development, 2010.

6. Eang MT et al. Early detection of tuberculosis through community-based active case finding in Cambodia. BMC Public Health, 2012, 12:469. doi:10.1186/1471-2458-12-469 pmid:22720878

7. Addressing Poverty in TB Control Options for National TB Control Programmes. Geneva, World Health Organization, 2005 (http:// whqlibdoc.who.int/hq/2005/WHO_HTM_TB_2005.352.pdf, accessed 30 September 2012).

8. Chan-yeung M et al. Socio-demographic and geographic indicators and distribution of tuberculosis in Hong Kong: a spatial analysis. The International Journal of Tuberculosis and Lung Disease, 2005, 9:1320-1326. pmid:16466053 
9. Jia ZW et al. Spatial analysis of tuberculosis cases in migrants and permanent residents, Beijing, 2000-2006. Emerging Infectious Diseases, 2008, 14:1413-1419. doi:10.3201/1409.071543 pmid: 18760008

10. Pang PT-T, Leung CC, Lee SS. Neighbourhood risk factors for tuberculosis in Hong Kong. The International Journal of Tuberculosis and Lung Disease, 2010, 14:585-592. pmid:20392351

11. Borgdorff MW. New measurable indicator for tuberculosis case detection. Emerging Infectious Diseases, 2004, 10:1523-1528. doi:10.3201/eid1009.040349 pmid:15498151

12. Report Second National Tuberculosis Prevalence Survey, Cambodia, 2011. Phnom Penh, National Center for Tuberculosis and Leprosy Control, 2012 (http://www.cenat.gov.kh/sites/
default/files/files/documents/files/Second\%20National\%20 Tuberculosis\%20Prevalence\%20Survey\%2C\%20Cambodia\%20 2011.pdf, accessed 3 January 2013).

13. Mangtani $P$ et al. Socioeconomic deprivation and notification rates for tuberculosis in London during 1982-91. BMJ (Clinical Research Ed.), 1995, 310:963-966. doi:10.1136/ bmj.310.6985.963 pmid:7728030

14. Spence DP et al. Tuberculosis and poverty. BMJ (Clinical Research Ed.), 1993, 307:759-761. doi:10.1136/bmj.307.6907.759 pmid:8219945

15. Uplekar M et al. Gender and tuberculosis control: towards a strategy for research and action. Geneva, World Health Organization, 1999 (http://whqlibdoc.who.int/hq/2000/WHO_CDS_TB_2000.280. pdf, accessed 30 September 2012). 